\title{
Anthocyanins Participate in the Protection of Wheat Seedlings against Cadmium Stress
}

\author{
O.Yu. ShOEVA ${ }^{1 *}$ and E.K. KhLESTKINA ${ }^{1,2}$ \\ ${ }^{1}$ Institute of Cytology and Genetics, Siberian Branch of the Russian Academy of Sciences, \\ Lavrentjeva Ave. 10, Novosibirsk, 630090 Russia \\ ${ }^{2}$ Novosibirsk State University, Pirogova St. 2, Novosibirsk, 630090 Russia
}

(Received 29 March 2017; Accepted 26 June 2017;

Communicated by A. Aniol)

\begin{abstract}
Due to anthropogenic activity, the environment is contaminated with high levels of cadmium, which is a dangerous heavy metal. At very low concentrations, cadmium is bioaccumulative and toxic to animals and plants, generating reactive oxygen species (ROS) that are destructive to cells of organisms. Anthocyanin pigments are natural antioxidants produced in various plant tissues and play a protective role under different environments. In the present study, the putative role of anthocyanins that accumulate in the grains and shoots of bread wheat (Triticum aestivum L.) in response to cadmium-induced toxicity ( 25 and $50 \mu \mathrm{M}$ $\mathrm{CdCl}_{2}$ ) was studied at the seedling stage. For this purpose, a set of near-isogenic lines carrying different alleles of the $P p$ (purple pericarp) and $R c$ (red coleoptile) genes was used. The lines responded differently to $\mathrm{Cd}$ treatment. The observed changes in anthocyanin metabolism under stress conditions were dependent on the alleles of the $R c$ genes that determine coleoptile pigmentation and on $\mathrm{CdCl}_{2}$ concentration. In less-colored line carrying the $R c-A 1$ allele, the antioxidant system was unable to fully cope with oxidative stress and thus induced the synthesis of additional antioxidants, whereas in the most tolerant lines, which have darkpurple coleoptile pigmentation predetermined by $R c-A 1+R c-D 1$, the level of anthocyanins in the coleoptiles was independent of stress. A protective role of anthocyanins presented in the coleoptiles of wheat seedlings was observed under moderate Cd stress $(25 \mu \mathrm{M})$, whereas anthocyanins seemed to be ineffective as protective compounds under heavier stress.
\end{abstract}

Keywords: Triticum aestivum L., heavy metal, intensity of stress, root and shoot lengths

\section{Introduction}

Heavy metals are major environmental pollutants. Heavy metals enter the soil, water, and air as a result of both natural processes (weathering of rocks, volcanic activity) and human economic activity (mining, metallurgy, chemical industry, transport, mineral fertilizing) (Kulaeva and Tsyganov 2011). Among heavy metals, $\mathrm{Cd}$ is one of the most dangerous and widespread contaminant elements. It is highly toxic to plants and animals even at very low concentrations.

\footnotetext{
*Corresponding author; E-mail: olesya_ter@bionet.nsc.ru; Phone: +7(383)363-49-63; Fax: +7(383)333-12-78
} 
In plants, one of the harmful effects of $\mathrm{Cd}$ is the generation of reactive oxygen species (ROS), such as superoxide radical, hydrogen peroxide, and hydroxyl radical, that cause oxidative stress and disrupt the biochemical and physiological functions of cells (Azevedo et al. 2012).

To cope with ROS, plants have an antioxidant protective system. This system includes both enzymatic antioxidants, such as superoxide dismutase, glutathione reductase, catalase, glutathione and ascorbate peroxidases, that detoxify $\mathrm{H}_{2} \mathrm{O}_{2}$ and non-enzymatic antioxidants, such as ascorbate and glutathione, vitamins, flavonoids, alkaloids, and carotenoids (Ahmad et al. 2010). Among the non-enzymatic antioxidants, colored flavonoid compounds anthocyanins can also be considered. These compounds demonstrate a powerful antioxidant capacity that is almost four times greater than that of ascorbic acid and $\alpha$-tocopherol (Bors et al. 1994; Wang et al. 1997). The intensification of anthocyanin biosynthesis has been observed under different types of stresses, including heavy metal stress (Chalker-Scott 1999; Landi et al. 2015b). Anthocyanins can chelate some heavy metal ions, preventing oxidative stress and the translocation of the harmful heavy metals ions (Landi 2015a).

Bread wheat can accumulate anthocyanins in vegetative and generative organs (Khlestkina 2012), but the protective functions of the pigments in wheat tissues with respect to the cadmium-induced toxicity have not been studied. The goal of the present study was to characterize the putative protective role of anthocyanins of wheat grains and coleoptiles in response to $\mathrm{Cd}$ stress. To investigate this issue, a precise genetic model - a set of nearisogenic lines (NILs) differing by the allelic state of genes conferring purple grain $(P p)$ and coleoptile $(R c)$ color - was used.

\section{Materials and Methods}

\section{Plant material}

The following wheat genotypes differing in anthocyanin pigmentation of the pericarp and coleoptile were used: (1) cultivar 'Saratovskaya 29' ('S29'), which has a light-red coleoptile controlled by the dominant $R c-A 1$ allele and a non-colored pericarp predetermined by a combination of $P p$ alleles $-P p-A 1+p p-D 1+p p 3$; (2) near-isogenic lines (NILs) developed in the 'S29' background (Arbuzova et al. 1998) that have an additional $R c$ gene $(R c-D 1)$ providing dark-red coleoptile pigmentation and two dominant complementary genes $(P p-D 1$ and $P p 3)$ that determine the dark-purple pigmentation of the pericarp, inherited from cultivars 'Purple Feed' (PF) and 'Purple' (P). These lines, designated i:S29Pp-A1Pp-D1Pp3 ${ }^{\mathrm{PF}}$ ('iPF') and $\mathrm{i}: \mathrm{S} 29 P p-A 1 P p-D 1 P p 3^{\mathrm{P}}$ ('iP'), respectively, have been previously characterized using microsatellite markers (Tereshchenko et al. 2012; Gordeeva et al. 2015) and currently are maintained in the ICG collection "GenAgro". 


\section{Experimental procedure}

One-day-old germinated seedlings growing on filter paper at $20^{\circ} \mathrm{C}$ under a 12 -hour photoperiod in a "Rubarth Apparate" growth chamber (RUMED GmbH, Laatzen, Germany) were exposed to 0 (control), 25 or $50 \mu \mathrm{M} \mathrm{CdCl}_{2}$. From the $3^{\text {rd }}$ to the $7^{\text {th }}$ day after germination, the lengths of shoots and roots were measured. The experiment was performed in triplicate for each concentration and each genotype, with ten seedlings per replicate. Seeds produced in the same year and under the same climatic conditions were used.

\section{Anthocyanin extraction and measurement}

For anthocyanin content evaluation in coleoptile of seedlings, three NILs were grown in the same conditions as described above (20 seedlings were grown per replicate). Every day from the $3^{\text {rd }}$ to the $7^{\text {th }}$ day after germination, coleoptiles from four seedlings were pooled and homogenized in $1 \% \mathrm{HCl} /$ methanol $(5 \mathrm{ml}$ per $1 \mathrm{~g}$ of plant material). The mixture was incubated at $4{ }^{\circ} \mathrm{C}$ for 24 hours and was centrifuged at $10,000 \mathrm{~g}$ for $30 \mathrm{~min}$ at $4{ }^{\circ} \mathrm{C}$. The collected supernatant was used to measure the relative anthocyanin content at a $530-\mathrm{nm}$ wavelength (OD530) using a SmartSpec Plus spectrophotometer (Bio-Rad, USA).

The assessment of anthocyanin content in the grain was performed at the wax-ripeness stage. For each genotype, anthocyanins were extracted in triplicate. Seeds coats for each replicate were peeled from the grains of one spike. The anthocyanin extraction procedure was the same as that described above.

\section{Statistical analysis}

The data were recorded as the mean value \pm standard deviation $(n=3)$. Significant differences between treated and control samples of the NILs were assessed using the non-parametric Mann-Whitney $U$-test, with $p \leq 0.05$ being significant. Changes in root length were calculated as follows:

$$
\left(\frac{\text { root length under treatment }}{\text { root length without treatment }}-1\right) \times 100 \% \text {. }
$$

The changes in shoot length and OD530 values of anthocyanin extracts were calculated in the same manner. The non-parametric Kruskal-Wallis $H$-test was used to determine the influence of the factor 'color' on the changes of growth parameters of wheat seedlings. Spearman's rank correlation coefficients between parameters were calculated using the software Statistica v. 6.1 (StatSoft, Inc.).

\section{Results}

\section{Changes in shoot and root length}

Under control conditions, the root lengths did not differ between the lines. The longest shoots were observed in ' $\mathrm{iP}$ ' (from the $5^{\text {th }}$ to the $7^{\text {th }}$ day after germination) and in 'iPF' (on the $6^{\text {th }}$ day) (Table 1). Cd affected morphological parameters. The lengths of shoots and 

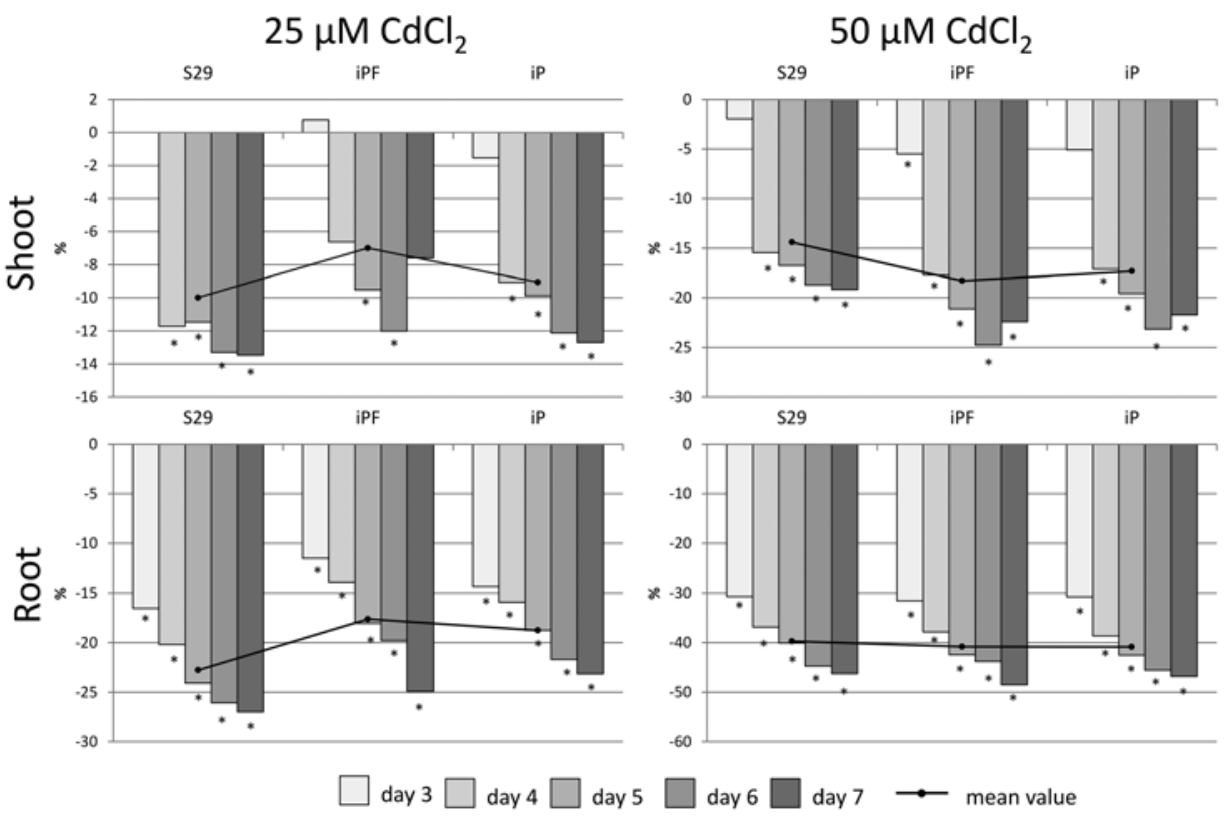

Figure 1. Changes in shoot and root length of 'S29', 'iPF' and 'iP' in response to cadmium chloride exposure relative to those of the control $(\%)$. *Significant differences between stressed and control samples at $p \leq 0.05$ (U-test)

roots of the lines decreased significantly after 25 and $50 \mu \mathrm{M} \mathrm{CdCl} 2$ treatments in comparison with those of the untreated control samples (Fig. 1).

The level of change varied among lines. After $25 \mu \mathrm{M} \mathrm{CdCl}_{2}$, the shoot length of ' $\mathrm{S} 29$ ' changed from 0.0 to $-13.6 \%$ ( $-10.0 \%$ on average throughout the experiment); the shoot length of ' $\mathrm{iPF}$ ' and ' $\mathrm{iP}$ ' varied from +0.8 to $-12.0 \%(-7.0 \%)$ and from -1.5 to $-12.7 \%$ $(-9.1 \%)$, respectively. A reduction in root length after $25 \mu \mathrm{M} \mathrm{CdCl}_{2}$ occurred from -16.5 to $-27.0 \%(-22.8 \%)$ in 'S29', whereas in 'iPF' and ' $\mathrm{PP}$ ', root length varied from -11.5 to $-24.9 \%(-17.6 \%)$ and from -14.3 to $-23.1 \%(-18.8 \%)$, respectively.

After $50 \mu \mathrm{M} \mathrm{CdCl}_{2}$ treatment, the shoot length changed from -2.0 to $-19.2 \%(-14.4 \%)$ in ' $\mathrm{S} 29$ ', from -5.5 to $-24.8 \%(-18.3 \%)$ in 'iPF', and from -5.1 to $-23.1 \%(-17.3 \%)$ in 'iP'. The root length changed from -30.7 to $-46.3 \%(-39.7 \%)$ in 'S29', from -31.6 to $-48.5 \%(-40.8 \%)$ in 'iPF', and from -30.8 to $-46.8 \%(-40.9 \%)$ in 'iP'.

The differences between the lines were more drastic after exposure to $25 \mu \mathrm{M} \mathrm{CdCl}_{2}$ than after exposure to $50 \mu \mathrm{M} \mathrm{CdCl}_{2}$.

There were negative correlations between the shoot length of control plants and the extent of the shoot length reduction under Cd stress $\left(r_{s}=-0.60, p \leq 0.05\right)$ and between the root length of control plants and extent of the root length reduction $\left(r_{s}=-0.45\right.$, $p \leq 0.05$ ). 


\begin{tabular}{|c|c|c|c|c|c|c|c|c|c|c|c|c|c|c|c|c|}
\hline \multirow{3}{*}{$\begin{array}{l}\overrightarrow{0} \\
0 \\
0 \\
\sum_{3}^{N} \\
i\end{array}$} & $\therefore$ & 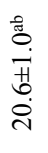 & 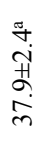 & $\begin{array}{c}\tilde{D} \\
\dot{N} \\
+ \\
+ \\
\infty \\
\infty\end{array}$ & 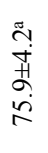 & 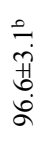 & $\begin{array}{l}\stackrel{\tilde{a}}{0} \\
\ddot{H} \\
\ddot{\sim}\end{array}$ & 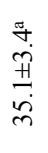 & $\begin{array}{l}\text { ă } \\
\dot{+} \\
\dot{H} \\
\dot{y} \\
\dot{y}\end{array}$ & $\begin{array}{l}\tilde{\infty} \\
\infty \\
1 \\
0 \\
\infty \\
\dot{+} \\
\dot{+}\end{array}$ & 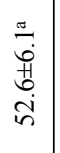 & 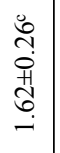 & $\begin{array}{l}\stackrel{0}{7} \\
\stackrel{0}{0} \\
\stackrel{1}{n} \\
\stackrel{n}{-}\end{array}$ & $\begin{array}{c}0 \\
= \\
\dot{0} \\
\text { H } \\
\stackrel{n}{-}\end{array}$ & 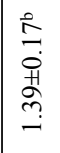 & 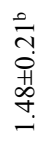 \\
\hline & $\stackrel{w}{=}$ & 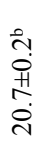 & $\frac{\stackrel{n}{*}}{\frac{H}{n}}$ & $\begin{array}{l}\tilde{r} \\
\dot{H} \\
2 \\
\dot{0} \\
i n\end{array}$ & 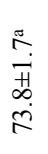 & $\begin{array}{l}\tilde{a} \\
\text { i } \\
\text { H } \\
\text { ? } \\
\sigma\end{array}$ & 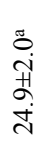 & $\begin{array}{l}\text { त̃ } \\
\text { तै } \\
\text { ஸे } \\
\text { లn }\end{array}$ & $\begin{array}{l}\tilde{\infty} \\
\dot{p} \\
\dot{y} \\
\dot{\gamma} \\
\dot{f}\end{array}$ & $\begin{array}{l}\stackrel{\tilde{n}}{0} \\
\tilde{D} \\
\stackrel{+}{+} \\
\vec{f}\end{array}$ & $\begin{array}{l}\tilde{\infty} \\
\dot{n} \\
\pm \\
\ddot{n} \\
\tilde{n}\end{array}$ & $\begin{array}{l}0 \\
\hat{6} \\
0 \\
0 \\
0 \\
0 \\
\dot{1} \\
i\end{array}$ & $\begin{array}{l}0 \\
0 \\
0 \\
0 \\
+ \\
+ \\
0 \\
-\end{array}$ & $\begin{array}{c}0 \\
0 \\
0 \\
0 \\
+1 \\
+ \\
- \\
-i\end{array}$ & 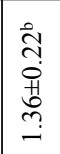 & 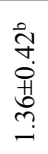 \\
\hline & సे & 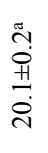 & $\begin{array}{l}\text { ă } \\
\text { ò } \\
\stackrel{+}{n} \\
\text { ñ }\end{array}$ & 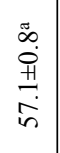 & $\begin{array}{l}\stackrel{\infty}{\infty} \\
\stackrel{+}{n} \\
\stackrel{n}{n} \\
\end{array}$ & $\begin{array}{l}\text { 品 } \\
\text { m } \\
\text { nू. }\end{array}$ & $\begin{array}{l}\tilde{D} \\
\stackrel{+}{ } \\
\mathbb{H} \\
\dot{d}\end{array}$ & $\begin{array}{l}\frac{\pi}{m} \\
+1 \\
\dot{0} \\
\dot{n}\end{array}$ & $\begin{array}{l}\stackrel{\tilde{m}}{?} \\
\stackrel{+}{+} \\
\stackrel{H}{+} \\
\dot{f}\end{array}$ & $\begin{array}{l}\tilde{a} \\
i \\
n \\
m \\
\infty \\
+ \\
+\end{array}$ & $\begin{array}{l}\tilde{0} \\
\dot{n} \\
H \\
\dot{n} \\
\dot{n}\end{array}$ & 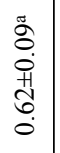 & $\begin{array}{l}\tilde{0} \\
0 \\
0 \\
0 \\
0 \\
0 \\
0 \\
0\end{array}$ & $\begin{array}{c}\tilde{n} \\
\stackrel{0}{0} \\
\stackrel{+}{+} \\
\stackrel{+}{n} \\
0\end{array}$ & $\mid \begin{array}{l}0 \\
0 \\
0 \\
0 \\
11 \\
0 \\
0 \\
0\end{array}$ & $\begin{array}{l}\text { 苂 } \\
0 \\
0 \\
0 \\
0 \\
0 \\
0\end{array}$ \\
\hline \multirow{3}{*}{ 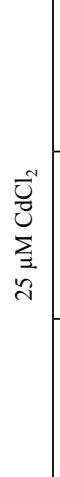 } & $\therefore$ & 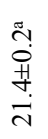 & $\begin{array}{l}\text { तै } \\
\text { सु } \\
\dot{\vec{\nabla}}\end{array}$ & $\begin{array}{l}\stackrel{0}{0} \\
\stackrel{0}{+} \\
+ \\
\dot{0}\end{array}$ & $\begin{array}{l}\stackrel{0}{m} \\
\stackrel{+}{i} \\
\stackrel{+}{0} \\
\dot{\infty}\end{array}$ & 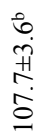 & $\begin{array}{l}\frac{\tilde{\sigma}}{\dot{H}} \\
\frac{\text { H }}{m}\end{array}$ & 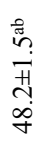 & 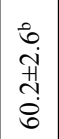 & 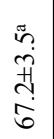 & $\begin{array}{l}\tilde{0} \\
\stackrel{+}{+} \\
+ \\
0 \\
\dot{0}\end{array}$ & $\begin{array}{l}\stackrel{0}{0} \\
\stackrel{0}{0} \\
\dot{1} \\
0 \\
\dot{i}\end{array}$ & $\begin{array}{l}\stackrel{0}{0} \\
\stackrel{0}{0} \\
\stackrel{1}{2} \\
\stackrel{2}{i}\end{array}$ & $\begin{array}{c}0 \\
0 \\
0 \\
0 \\
0 \\
0 \\
0 \\
i \\
\text { in }\end{array}$ & 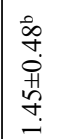 & 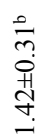 \\
\hline & $\stackrel{\omega}{=}$ & $\begin{array}{l}\text { ơ. } \\
\text { से } \\
\text { ते } \\
\text { ते }\end{array}$ & $\begin{array}{l}\stackrel{ }{N} \\
\stackrel{+}{+} \\
\stackrel{\text { Ij }}{\text { I }}\end{array}$ & $\begin{array}{c}0 \\
\infty \\
i \\
i \\
n \\
i \\
b\end{array}$ & 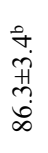 & $\begin{array}{l}\stackrel{i}{n} \\
\text { H } \\
\hat{H} \\
\infty \\
0 \\
0\end{array}$ & 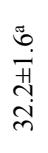 & $\begin{array}{l}\stackrel{0}{0} \\
\text { in } \\
\dot{H} \\
\dot{8}\end{array}$ & $\begin{array}{l}\text { aे } \\
\text { ì } \\
\text { ț } \\
\text { b }\end{array}$ & $\begin{array}{l}\text { oू } \\
0 \\
+1 \\
m \\
0 \\
0\end{array}$ & $\begin{array}{l}\tilde{0} \\
i \\
+ \\
a \\
i n \\
i\end{array}$ & $\begin{array}{l}\stackrel{0}{n} \\
\stackrel{0}{0} \\
\stackrel{+}{m} \\
\stackrel{2}{c} \\
i\end{array}$ & 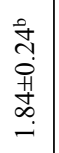 & $\begin{array}{c}i n \\
i \\
0 \\
+1 \\
\infty \\
-i \\
-1\end{array}$ & $\begin{array}{l}\text { ñ } \\
n \\
0 \\
+1 \\
0 \\
\\
\end{array}$ & 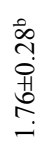 \\
\hline & స్టे & 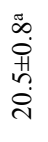 & $\begin{array}{l}\stackrel{\Xi}{0} \\
\stackrel{H}{+} \\
\infty \\
\infty \\
\infty \\
\infty\end{array}$ & 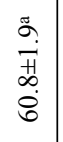 & 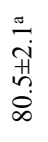 & $\begin{array}{l}\frac{\pi}{m} \\
\dot{p} \\
\infty \\
\alpha \\
\alpha\end{array}$ & 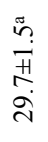 & 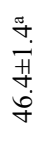 & \begin{tabular}{l}
$\tilde{a}$ \\
\multirow{n}{*}{} \\
$\dot{n}$ \\
$i n$
\end{tabular} & 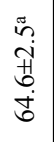 & $\begin{array}{l}\frac{\pi}{n} \\
\dot{n} \\
\dot{0} \\
\dot{n}\end{array}$ & $\begin{array}{l}\ddot{2} \\
\ddot{0} \\
\dot{0} \\
+1 \\
0 \\
\dot{0}\end{array}$ & 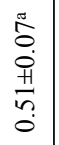 & $\begin{array}{l}\tilde{n} \\
\tilde{0} \\
\ddot{0} \\
n \\
\tilde{n} \\
0\end{array}$ & $\begin{array}{l}\tilde{D} \\
0 \\
0 \\
0 \\
1 \\
\tilde{N} \\
\tilde{0} \\
0\end{array}$ & 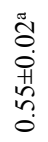 \\
\hline \multirow{3}{*}{$\begin{array}{l}\overrightarrow{0} \\
\stackrel{\Xi}{0} \\
\dot{0}\end{array}$} & $\therefore$ & 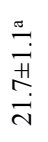 & $\frac{\frac{a}{\sigma}}{\stackrel{H}{f}}$ & 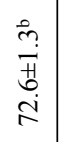 & $\begin{array}{l}\dot{b} \\
\frac{1}{H} \\
\infty \\
\infty \\
\infty\end{array}$ & 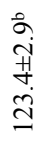 & 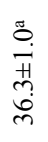 & $\begin{array}{l}\frac{\pi}{\sim} \\
\frac{H}{m} \\
\text { in }\end{array}$ & 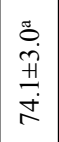 & $\begin{array}{l}\tilde{n} \\
\tilde{n} \\
\ddot{H} \\
\ddot{\infty}\end{array}$ & 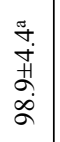 & $\begin{array}{l}\stackrel{0}{+} \\
\stackrel{+}{0} \\
\stackrel{+}{y} \\
\stackrel{i}{i}\end{array}$ & 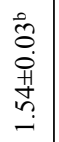 & 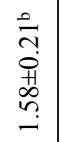 & $\begin{array}{l}0 \\
\tilde{n} \\
0 \\
0 \\
0 \\
0 \\
0 \\
\end{array}$ & 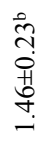 \\
\hline & $\stackrel{L}{=}$ & $\begin{array}{l}\frac{\pi}{J} \\
\frac{\vec{H}}{\Delta} \\
\stackrel{\vec{\lambda}}{ }\end{array}$ & 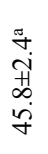 & 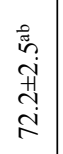 & $\begin{array}{l}0 \\
\stackrel{n}{\pi} \\
+ \\
0 \\
\infty \\
\infty\end{array}$ & 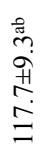 & 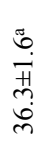 & 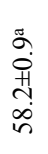 & $\begin{array}{l}\tilde{g} \\
\dot{H} \\
\stackrel{H}{r} \\
\dot{I}\end{array}$ & 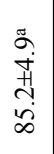 & 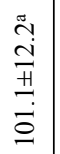 & $\begin{array}{l}\stackrel{2}{a} \\
\overrightarrow{0} \\
+ \\
\stackrel{+}{N} \\
i\end{array}$ & 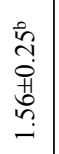 & 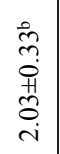 & 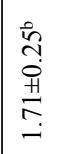 & 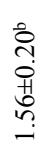 \\
\hline & సે & 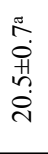 & 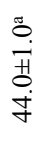 & 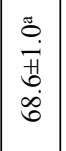 & $\begin{array}{l}\frac{\tilde{g}}{\mathrm{~g}} \\
\text { से } \\
\text { à }\end{array}$ & 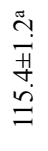 & 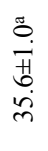 & $\begin{array}{l}\tilde{n} \\
\text { on } \\
+ \\
\text { n. } \\
\infty \\
n\end{array}$ & 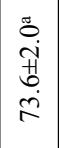 & 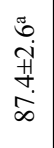 & $\begin{array}{l}\stackrel{\ddot{n}}{-} \\
\overrightarrow{+} \\
\stackrel{\alpha}{\alpha}\end{array}$ & $\begin{array}{l}\tilde{n} \\
0 \\
0 \\
0 \\
n \\
n \\
0\end{array}$ & 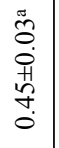 & \begin{tabular}{l}
$\tilde{\infty}$ \\
0 \\
0 \\
0 \\
+1 \\
0 \\
\multirow{0}{0}{} \\
0
\end{tabular} & 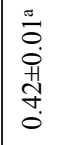 & 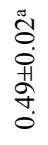 \\
\hline$\tilde{\theta}$ & & $m$ & $\nabla$ & $n$ & 0 & $n$ & $m$ & $\nabla$ & in & 0 & $r$ & $m$ & $\nabla$ & in & 0 & $r$ \\
\hline$\frac{\pi}{5}$ & & & & 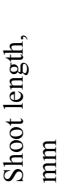 & & & & & 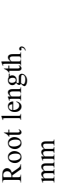 & & & & & 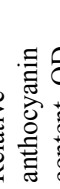 & & \\
\hline
\end{tabular}




\section{Changes in anthocyanin content}

Under non-stress conditions, 'S29' (a carrier of the $R c$ - $A 1$ gene) had approximately 4-fold lower OD530 values (relative anthocyanin content) in coleoptiles in comparison with those of intensively colored NILs ('iPF' and ' $\mathrm{PP}$ ' - the carriers of the $R c-A 1+R c-D 1$ genes) (Table 1). 'iPF' and 'iP coleoptiles had similar anthocyanin contents (Table 1), while their grains contained significantly different amounts of these pigmented substances. The OD530 of 'iPF' grain extracts was $2.3 \pm 0.2$, while that of ' $\mathrm{iP}$ ' was $3.5 \pm 0.3$. Anthocyanins were absent in the grains of 'S29'.

Under cadmium chloride exposure, the anthocyanin level in coleoptiles changed differently in different lines (Fig. 2). A statistically significant increase in anthocyanin content in the coleoptile was recorded in ' $\mathrm{S} 29$ ' on the $6^{\text {th }}$ day under $25 \mu \mathrm{M} \mathrm{CdCl}_{2}$ and on the

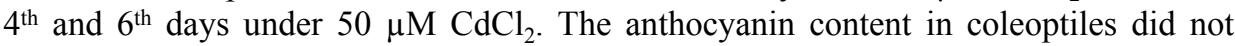
change in ' $\mathrm{iPF}$ ' under both concentrations of cadmium chloride; however, the anthocyanin content increased in ' $\mathrm{iP}$ ' (on the $4^{\text {th }}$ day) under $25 \mu \mathrm{M} \mathrm{CdCl}_{2}$ but decreased (on the $3^{\text {rd }}$ day) under $50 \mu \mathrm{M} \mathrm{CdCl}_{2}$ (Fig. 2).
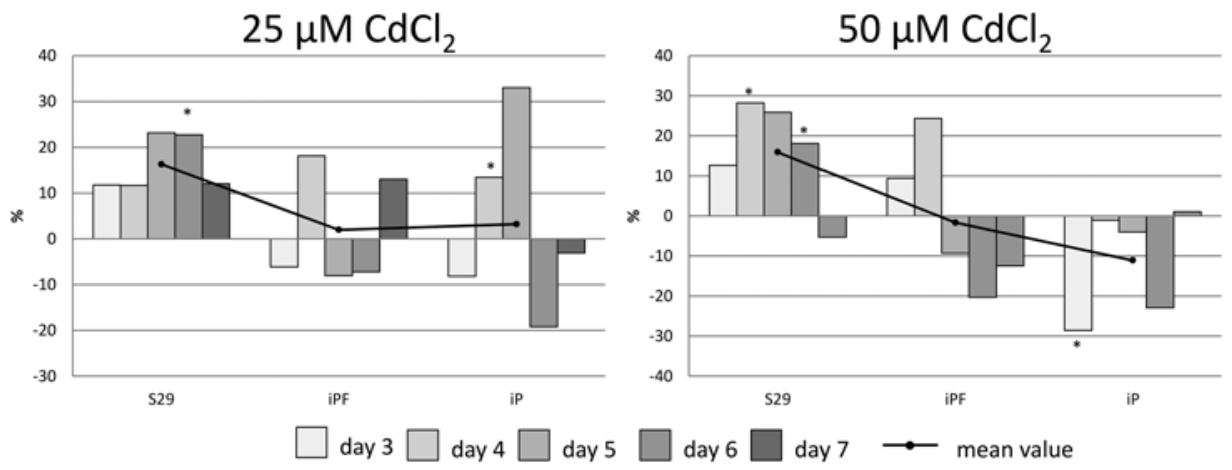

Figure 2. Changes in anthocyanin contents in coleoptiles of 'S29', 'iPF' and 'iP' in response to cadmium chloride exposure relative to those of the control (\%). *Significant differences between stressed and control samples at $p \leq 0.05$ (U-test)

\section{Influence of the coleoptile and grain color on growth parameters}

One-way ANOVA on ranks was run to evaluate the effects of color on growth parameter changes in wheat seedlings under Cd stress. As summarized in Table 2, two groups of samples ('non-colored' vs 'colored') were significantly different with respect to anthocyanin content in coleoptiles and grains under control conditions, in changes in shoot length under $25 \mu \mathrm{M} \mathrm{CdCl}_{2}$ and in changes in anthocyanin content in coleoptiles under 50 $\mu \mathrm{M} \mathrm{CdCl}_{2}$. On the other hand, the other parameters, including root and shoot length under control conditions, were not different. 
Table 2. Kruskal-Wallis $H$-test analysis. Group size: 'non-colored' group has five values of traits of 'S29', 'colored' group has ten values of traits of 'iPF' and 'iP'; degrees of freedom (df): 1

\begin{tabular}{|c|c|c|c|c|c|}
\hline Trait & Group & Group size & $\begin{array}{l}\text { Sum } \\
\text { of ranks }\end{array}$ & $H$ & $p$-Value \\
\hline \multicolumn{6}{|c|}{$0 \mu \mathrm{M} \mathrm{CdCl}_{2}$} \\
\hline \multirow{2}{*}{$\begin{array}{l}\text { Anthocyanin content in the } \\
\text { coleoptile (in control) }\end{array}$} & 'non-colored' & 5 & 15 & \multirow{2}{*}{9.38} & \multirow{2}{*}{0.0022} \\
\hline & 'colored' & 10 & 105 & & \\
\hline \multirow{2}{*}{ Anthocyanin content in the grains } & 'non-colored' & 5 & 15 & \multirow{2}{*}{10.5} & \multirow{2}{*}{0.0012} \\
\hline & 'colored' & 10 & 105 & & \\
\hline \multirow{2}{*}{ Root length (in control) } & 'non-colored' & 5 & 40 & \multirow{2}{*}{0.00} & \multirow{2}{*}{1.0000} \\
\hline & 'colored' & 10 & 80 & & \\
\hline \multirow{2}{*}{ Shoot length (in control) } & 'non-colored' & 5 & 35 & \multirow{2}{*}{0.38} & \multirow{2}{*}{0.5403} \\
\hline & 'colored' & 10 & 85 & & \\
\hline \multicolumn{6}{|c|}{$25 \mu \mathrm{M} \mathrm{CdCl}_{2}$} \\
\hline \multirow{2}{*}{$\begin{array}{l}\text { Changes in the anthocyanin content } \\
\text { in the coleoptile }\end{array}$} & 'non-colored' & 5 & 51 & \multirow{2}{*}{1.82} & \multirow{2}{*}{0.1779} \\
\hline & 'colored' & 10 & 69 & & \\
\hline \multirow{2}{*}{ Changes in root length } & 'non-colored' & 5 & 25 & \multirow{2}{*}{3.38} & \multirow{2}{*}{0.0662} \\
\hline & 'colored' & 10 & 95 & & \\
\hline \multirow{2}{*}{ Changes in shoot length } & 'non-colored' & 5 & 24 & \multirow{2}{*}{3.85} & \multirow{2}{*}{0.0498} \\
\hline & 'colored' & 10 & 96 & & \\
\hline \multicolumn{6}{|c|}{$50 \mu \mathrm{M} \mathrm{CdCl}_{2}$} \\
\hline \multirow{2}{*}{$\begin{array}{l}\text { Changes in the anthocyanin content } \\
\text { in the coleoptile }\end{array}$} & 'non-colored' & 5 & 58 & \multirow{2}{*}{4.86} & \multirow{2}{*}{0.0275} \\
\hline & 'colored' & 10 & 62 & & \\
\hline \multirow{2}{*}{ Changes in root length } & 'non-colored' & 5 & 44 & \multirow{2}{*}{0.24} & \multirow{2}{*}{0.6242} \\
\hline & 'colored' & 10 & 76 & & \\
\hline \multirow{2}{*}{ Changes in shoot length } & 'non-colored' & 5 & 53 & \multirow{2}{*}{2.54} & \multirow{2}{*}{0.1113} \\
\hline & 'colored' & 10 & 67 & & \\
\hline
\end{tabular}

Overall, the data demonstrated that the factor 'dark-purple color' had a significant impact on changes in growth parameters of wheat seedlings under exposure to low doses of cadmium chloride.

\section{Correlations between anthocyanin levels and growth parameters}

Moderate negative correlations were observed between anthocyanin contents in the coleoptiles of control plants and reductions of shoot $\left(r_{s}=-0.595, p \leq 0.05\right)$ and root $\left(r_{s}=-0.600, p \leq 0.05\right)$ length under exposure to $25 \mu \mathrm{M} \mathrm{CdCl}$. No correlation was ob- 
served between the parameters under $50 \mu \mathrm{M} \mathrm{CdCl}_{2}$ or between the anthocyanin content in grains and changes in growth parameters.

\section{Discussion}

\section{Genotype- and stress-dependent regulation of anthocyanin biosynthesis}

Under cadmium stress, the accumulation of anthocyanins has been observed in different plant species. For example, enhanced anthocyanin content has been demonstrated in the leaves of Lathyrus maritimus grown in soil polluted with Cd (Maslennikov 2013) as well as in the seedlings of Secale cereale (Maslennikov et al. 2013) and Oryza sativa (Roychoudhury et al. 2012) under Cd stress. Under conditions of environmental cadmium pollution, positive correlations between $\mathrm{Cd}$ and anthocyanin content in the needles of Picea abies as well as the leaves of Tilia cordata, Taraxacum officinale and Plantago major have been established (Tchoupakhina et al. 2012). Cadmium-induced anthocyanin accumulation and increased transcript abundance of related genes encoding chalcone synthase (CHS) and dihydroflavonol reductase (DFR) have been shown in the aquatic fern Azolla imbricata (Dai et al. 2006, 2012a). The induced anthocyanins demonstrate powerful antioxidant ability against various free radicals as well as $\mathrm{Cd}^{2+}$ chelation ability (Dai et al. 2008, 2012b). In the leaves of Populus deltoides, increasing anthocyanin concentration and transcription of the related genes encoding chalcone isomerase $(\mathrm{CHI})$, flavanone 3-hydroxylase (F3H), glycosyltransferase (UFGT) and DFR have been observed under $50 \mu \mathrm{M} \mathrm{CdCl}_{2}$, whereas under higher Cd concentration $(100$ and $150 \mu \mathrm{M})$, the anthocyanin content and gene transcription decrease (Zhang et al. 2014).

In the present study, the relationship between anthocyanin biosynthesis and cadmium stress was investigated using the wheat NILs differing by grain and seedling coloration, which is predetermined by the $P p-1+P p 3$ and $R c-1$ genes, respectively (Khlestkina 2012). The three NILs respond differently to cadmium stress. In 'S29', which has lightred coleoptile coloration determined by $R c-A 1$, intensification of anthocyanin biosynthesis was observed under 25 and $50 \mu \mathrm{M} \mathrm{CdCl}_{2}$. In the ' $\mathrm{iPF}$ ' line, with intense anthocyanin pigmentation of the coleoptile controlled by $R c-A 1+R c-D 1$, anthocyanin content was not altered under either $\mathrm{CdCl}_{2}$ concentration. 'iP', which has the dominant alleles $R c-A 1+$ $R c-D 1$, like 'iPF' seems to be more sensitive to stress than is the sister line (the anthocyanin level in 'iP' increased under $25 \mu \mathrm{M}$ but decreased under $50 \mu \mathrm{M} \mathrm{CdCl}_{2}$ ) (Fig. 2). 'iPF' and 'iP' have Rc-D1 alleles from different donors (Gordeeva et al. 2015) and may have different $c i s$-regulatory elements. Thus, the response of anthocyanin biosynthesis to cadmium stress is dependent on the alleles of the $R c-1$ genes. The genotype-dependent response of anthocyanin metabolism has been observed under different types of stresses in different cultivars and NILs of diverse plant species (Maekawa et al. 2001; Daneshmand et al. 2010; Basu et al. 2010; Borghesi et al. 2011; Roychoudhury et al. 2012; Ploenlap and Pattanagul 2015).

The NILs ('S29', 'iPF', 'iP') exploited in the present study have been previously tested under cold, osmotic stress and ionizing radiation (Gordeeva et al. 2013, 2017; Shoeva et 
al. 2017), which allows comparing the effects of different environmental stresses on changes in anthocyanin content in the same genotypes. Under cold stress, the anthocyanin content decreased in light-red coleoptiles of 'S29' but increased in the purple coleoptiles of 'iPF' and 'iP' (Gordeeva et al. 2013). After seeds were irradiated, a decrease in anthocyanin concentration was reported in the coleoptiles of 'S29', whereas in 'iPF' and 'iP', the anthocyanin content did not change (Gordeeva et al. 2017). Under osmotic stress, the mode of response was similar to that under Cd treatment: a higher intensification of anthocyanin biosynthesis was observed in 'S29' (Shoeva et al. 2017). Such patterns of anthocyanin metabolism under different types of stresses suggest that the regulation of anthocyanin biosynthesisis stress-dependent, although common mechanisms can be involved in the response to osmotic and cadmium stresses.

\section{Protective role of anthocyanin pigmentation}

Comparison of the sensitivity of the NILs under stress conditions allows revealing the protective role of pigments under various stress conditions (Gordeeva et al. 2013; Shoeva et al. 2017). In the present study, it was shown that anthocyanins produced in coleoptiles protect wheat seedlings against moderate Cd stress $(25 \mu \mathrm{M})$. The more anthocyanins that are present in wheat seedlings in control conditions, the less reduction of shoot and root lengths under cadmium stress observed (Fig. 1, left part). The lines ranked by their tolerance to $25 \mu \mathrm{M} \mathrm{CdCl}_{2}$ are as follows: 'S29' < 'iP' < 'iPF' (Fig. 1, left part), and the more sensitive lines ('S29' and 'iP') demonstrate a tendency toward enhanced anthocyanin accumulation under moderate Cd stress (Fig. 2, left part) in contrast to the most tolerant 'iPF', which has stress-independent anthocyanin accumulations in the coleoptile (Fig. 2). ANOVA demonstrated that the factor 'dark-purple color' determined by the allele combination $R c-A 1+R c-D 1$ has a significant impact on the changes of growth parameters of wheat seedlings under $25 \mu \mathrm{M} \mathrm{CdCl}$. A similar role of this factor and associated genes was revealed for the same set of lines under osmotic stress (Shoeva et al. 2017). Furthermore, the most intense induction of anthocyanin synthesis was also observed in the coleoptiles of the most stress-sensitive line, suggesting similar mechanisms may be involved in $\mathrm{Cd}$ and osmotic stress responses in wheat seedlings. The observed protective role of the anthocyanins could be explained by their high antioxidant potential, while higher intensification of the anthocyanin biosynthesis in less-colored lines suggests that the antioxidant system is not able to fully cope with the oxidative stress, inducing the synthesis of additional antioxidants. The ability of anthocyanins to compensate for the deficiency of antioxidants was hypothesized by Zhu et al. (2013). In the work of those authors, the antioxidant systems of fully expanded and central leaves of Saccharum officinarum were compared under chilling stress. It was revealed that under a similar degree of chilling injury, the activity of antioxidant enzymes was higher in fully expanded leaves than in central ones, whereas anthocyanin concentration increased more in central leaves than in fully expanded ones.

In the present study, a protective role of anthocyanin pigments was observed under moderate $(25 \mu \mathrm{M}) \mathrm{CdCl}_{2}$ stress, whereas under heavier stress, the relationship between 
pigmentation and decreased root and shoot lengths was not identified (Table 2; Fig. 2, right part). The obtained results confirmed the earlier observation of the protective role of the anthocyanins of wheat coleoptiles under low doses of ionizing radiation but not under higher doses (Gordeeva et al. 2017). It seems that anthocyanins are more effective under low or moderate stress but are not powerful enough under heavy stress.

Overall, the changes in anthocyanin metabolism under stress conditions and the level of stress sensitivity/tolerance both depend on the following three factors: (i) the alleles of genes that control anthocyanin synthesis, (ii) the type of the stress and (iii) the intensity of stress.

\section{Acknowledgments}

We thank the RFBR (Grant No. 16-34-60052) for partial support of this study and Ms. Galina Generalova (ICG SB RAS) for technical assistance. Wheat growth in ICG Plant Growth Core Facility was supported by the ICG project (No. 0324-2016-0001).

\section{References}

Arbuzova, V.S., Maystrenko, O.I., Popova, O.M. 1998. Development of near-isogenic lines of the common wheat cultivar 'Saratovskaya 29'. Cereal Res. Commun. 26:39-46.

Ahmad, P., Jaleel C.A., Salem M.A., Nabi G., Sharma S. 2010. Roles of enzymatic and nonenzymatic antioxidants in plants during abiotic stress. Crit. Rev. Biotechnol. 30:161-175.

Azevedo, R.A., Gratão, P.L., Monteiro, C.C., Carvalho, R.F. 2012. What is new in the research on cadmiuminduced stress in plants? Food Energy Secur. 1:133-140.

Basu, S., Roychowdhury, A., Saha, P.P., Sengupta, D.N. 2010. Differential antioxidative responses of indica rice cultivars to drought stress. Plant Growth Regul. 60:51-59.

Borghesi, E., González-Miret, M.L., Escudero-Gilete, M.L., Malorgio, F., Heredia, F.J., Meléndez-Martínez, A.J. 2011. Effects of salinity stress on carotenoids, anthocyanins, and colour of diverse tomato genotypes. J. Agric. Food Chem. 59:11676-11682.

Bors, W., Mochel, C., Saran, M. 1994. Flavonoid antioxidants: Rate constants for reactions with oxygen radicals. Met. Enzymol. 234:420-429.

Chalker-Scott, L. 1999. Environmental significance of anthocyanins in plant stress responses. Photochem. Photobiol. 70:1-9.

Dai, L.P., Xiong, Z.T., Huang, Y., Li, M.J. 2006. Cadmium-induced changes in pigments, total phenolics, and phenylalanine ammonia-lyase activity in fronds of Azolla imbricata. Environ. Toxicol. 21:505-512.

Dai, L.P., Xiong, Z.T., Dong, X.J., NAN, X.Y., 2008. Antioxidant properties of anthocyanins in Azolla imbricata under cadmium stress. Acta Sci. Circum. 28:2089-2094.

Dai, L.P., Dong, X.J., Ma, H.H., 2012a. Molecular mechanism for cadmium-induced anthocyanin accumulation in Azolla imbricata. Chemosphere 87:319-325.

Dai, L.P., Dong, X.J., Ma, H.H., 2012b. Antioxidative and chelating properties of anthocyanins in Azolla imbricata induced by cadmium. Pol. J. Environ. Stud. 21:837-844.

Daneshmand, F., Arvin, M.J., Kalantari, K.M. 2010. Physiological responses to NaCl stress in three wild species of potato in vitro. Acta Physiol. Plant. 32:91-101.

Gordeeva, E.I., Shoeva, O.Y., Khlestkina, E.K. 2013. Cold stress response of wheat genotypes having different Rc alleles. Cereal Res. Commun. 41:519-526.

Gordeeva, E.I., Shoeva, O.Y., Khlestkina, E.K. 2015. Marker-assisted development of bread wheat near-isogenic lines carrying various combinations of Pp (purple pericarp) alleles. Euphytica. 203:469-476. 
Gordeeva, E.I., Shoeva, O.Yu., Yudina, R.S., Kukoeva, T.V., Khlestkina, E.K. 2017. Effect of seed pre-sowing gamma-irradiation treatment in bread wheat lines different by anthocyanin pigmentation. Cereal Res. Commun DOI: 10.1556/0806.45.2017.059.

Khlestkina, E.K. 2012. Genes determining coloration of different organs in wheat. Vavilov J. Genet. Breed. 16:202-216.

Kulaeva, O.A., Tsyganov, V.E. 2011. Molecular-genetic basis of cadmium tolerance and accumulation in higher plants. Russ. J. Genet. Appl. Res. 1:349-360.

Landi, M. 2015a. Can anthocyanins be part of the metal homeostasis network in plant? Am. J. Agric. Biol. Sci. 10:170-177.

Landi, M., Tattini, M., Gould, K.S. 2015b. Multiple functional roles of anthocyanins in plant-environment interactions. Environ. Exp. Bot. 119:4-17.

Maekawa, M., Sato, T., Kumagai, T., Noda, K. 2001. Differential responses to UV-B irradiation of three near isogenic lines carrying different purple leaf genes for anthocyanin accumulation in rice (Oryza sativa L.). Breed. Sci. 51:27-32.

Maslennikov, P.V. 2013. Reakciya antioksidantnoj sistemy chiny primorskoj (Lathyrus maritimus Bigel.) na dejstvie ionov kadmiya [Reaction of the antioxidant system of sea pea (Lathyrus maritimus Bigel.) on action of cadmium ions]. Aktualnye Problemy Gumanitarnyh i Estestvennyh Nauk [Actual problems of the Humanities and Natural Sciences $=$ Actual Probl. Humanit. Nat. Sci.]. 11:67-70. [in Russian]

Maslennikov, P.V., Velieva, E.T., Galyamova, Y.R. 2013. Rol nizkomolekulyarnyh antioksidantov v adaptacii ozimoj rzhi (Secale cereal L.) k toksicheskomu dejstviyu $\mathrm{CdCl}_{2}$ [Role of low molecular weight antioxidants in adaptation of winter rye (Secale cereal L.) to toxic level of $\left.\mathrm{CdCl}_{2}\right]$. Aktualnye Problemy Gumanitarnyh i Estestvennyh Nauk [Actual problems of the Humanities and Natural Sciences = Actual Probl. Humanit. Nat. Sci.]. 12:48-51. [in Russian]

Ploenlap, P., Pattanagul, W. 2015. Effects of exogenous abscisic acid on foliar anthocyanin accumulation and drought tolerance in purple rice. Biologia 70:915-921.

Roychoudhury, A., Basu, S., Sengupta, D.N. 2012. Antioxidants and stress-related metabolites in the seedlings of two indica rice varieties exposed to cadmium chloride toxicity. Acta Physiol. Plant. 34:835-847.

Shoeva, O.Yu., Gordeeva, E.I., Abuzova, V.S., Khlestkina, E.K. 2017. Anthocyanins participate in protection of wheat seedlings from osmotic stress. Cereal Res. Commun. 45:47-56.

Tereshchenko, O.Y., Gordeeva, E.I., Arbuzova, V.S., Börner, A., Khlestkina, E.K. 2012. The D genome carries a gene determining purple grain colour in wheat. Cereal Res. Commun. 40:334-341.

Tchoupakhina, G.N., Maslennikov, P.V., Skrypnik, L.N., Besserezhnova, M.I. 2012. Reaction of pigmental and antioxidant systems of plants on environmental pollution of Kaliningrad by motor transport emission. Tomsk State Univ. J. Biol. 2:171-185. [in Russian]

Wang, H., Cao, G., Prior, R.L. 1997. Oxygen radical absorbing capacity of anthocyanins. J. Agric. Food Chem. 45:304-309.

Zhang, F., Wan, X., Zheng, Y., Sun, L., Chen, Q., Guo, Y., Zhu, X., Liu, M. 2014. Physiological and related anthocyanin biosynthesis genes responses induced by cadmium stress in a new colored-leaf plant “Quanhong Poplar". Agroforest Syst. 88:343-355.

Zhu, J.J., Li, Y.R., Liao, J.X. 2013. Involvement of anthocyanins in the resistance to chilling-induced oxidative stress in Saccharum officinarum L. leaves. Plant Physiol. Biochem. 73:427-433. 